\title{
CLINICAL CORRESPONDENCE
}

\section{Cervicogenic headache caused by myofascial trigger points in the sternocleidomastoid: a case report}

\author{
JK Roth ${ }^{1}$, RS Roth ${ }^{2}$, JR Weintraub ${ }^{1}$ \& DG Simons ${ }^{3}$ \\ ${ }^{1}$ Michigan Head Pain and Neurological Institute, ${ }^{2}$ University of Michigan Health System, Department of Physical Medicine \& Rehabilitation, \\ Ann Arbor, MI, and ${ }^{3}$ Emory University, Department of Rehabilitation Medicine, Atlanta, GA, USA
}

Julie K. Roth, M.P.T., Michigan Head Pain \& Neurological Institute, 310

Professional Dr., Ann Arbor, MI 48104, USA. Tel. +1 734677 6000, fax +1 734

677 2422, e-mail jroth@mhni.com Received 12 October 2006, accepted 7 December

2006

Disturbances in the integrity of the upper cervical spine are now well known to be associated with head pain and can give rise to chronic daily headaches (1-3). Head pain that derives from cervical dysfunctions or disease is now contained under the category of cervicogenic headaches (4), which describes a heterogeneous group of disorders that commonly refer pain from structures in the cervical spine region (e.g. joints, muscle, nerve) to various regions in the head. The mechanisms that underlie head pain referred from the neck are not entirely understood. There is general consensus that the trigeminocervical complex, which is located in the lower brainstem and upper three cervical spinal segments and receives multiple afferent inputs from both the trigeminal nerve and cervical peripheral afferents, serves as the centre of convergence for the projection of perceived pain in the head that originates from cervical pain generators (1). However, this model may not explain all forms of cervicogenic pain, in particular myogenic referred head pain that derives from myofascial trigger points (MTrPs) of the cervical and upper back musculature $(5,6)$. An accurate diagnosis of the aetiology of cervicogenic headache can be challenging for the clinician due, in part, to the overlap of pain and associated symptoms between cervicogenic headache and primary headache $(3,7,8)$ and the multiple abnormalities of the cervical spine and musculature that can be a primary source of head pain (9-12).

Several authors have emphasized the role of anaesthetic nerve blockade, particularly directed to the occipital nerve and C2 spinal level, as the primary criterion for the diagnosis of cervicogenic headache $(1,3,13)$. The increasing attention to anaesthetic blockade as a diagnostic tool has paralleled the expansion of the use of interventional procedures for the treatment of chronic cervicogenic headache (14-16). Interventional diagnostic measures tend to follow from abnormal radiographic findings, such as spondylosis, disk disease or facet arthropathy $(1,9)$, and rely on patient report of diminished pain to anaesthetic challenge as a guide to diagnosis. Increasing reliance on nerve blockade as a diagnostic tool may encourage de-emphasis on the clinical examination of the musculature. Reliance on an interventional approach to the diagnosis of cervicogenic headache that targets joint arthropathies or possible nerve involvement tend to ignore the potential contribution of MTrPs or intersegmental motion restrictions as primary causes of cervicogenic headache $(10,17-19)$. Identification of the musculoskeletal dysfunctions requires a careful and informed clinical examination. Lack of familiarity with the characteristic myofascial referred pain patterns frequently leads to misdiagnosis and ineffectual headache interventions (10).

We report a case of a male with a 25-year history of chronic headaches despite an extensive course of medical evaluations and treatment. After a recent hospitalization and further interventional procedures to identify a cause of his pain, a careful physical examination of the cervical musculature identified MTrPs in the sternocleidomastoid (SCM) muscle as a primary source of his head pain. Physical therapy that focused on reducing the myofascial dysfunction and addressing perpetuating factors felt to be maintaining his MTrP activity brought about dramatic reduction in his pain within 6 weeks, which was maintained at 6 months. 


\section{Case report}

A 43-year old White male was referred to a head pain specialty clinic for evaluation of refractory headache. He described the onset of right-sided head pain at age 18, primarily in the right supraorbital region, with no history of significant cervical injury. Pain severity gradually increased and became constant by age 28 . At age 34 years, rightsided cervical and occipital pain began. Over the years, the patient saw many neurosurgeons, neurologists, pain specialists and a chiropractor and underwent five trials of physical therapy. The head pain was not accompanied by auras or prodrome. Aggravating factors included sleep deficit, weather changes, exercise and exertion, orgasm, neck movements, chewing, driving, Valsalva manoeuvres and coughing. Associated symptoms that fluctuated with head pain severity included decreased appetite, right-sided nasal stuffiness and drainage with conjunctival injection, tearing and right eye redness, cognitive impairment, inability to sleep more than 1-2 h continuously, fatigue and hearing a rushing, pulsating sound in the right ear. He rated his daily pain as moderate to severe and had a medical history of overuse of pain medication. Current medications included valdecoxib, gabapentin, hydrocodone and paracetamol.

Prior diagnostic work-up had included a cervical X-ray indicating 'very small' spurs posteriorly at the C4-5 and C5-6 levels, magnetic resonance imaging (MRI) revealing a mild left C5-6 disc protrusion without evidence of canal stenosis or nerve root involvement, and a computed tomographic (CT) scan of the brain was negative. Prior treatment had included multiple facet blocks at C3, C4 and C5 with temporary reduction of neck pain, and no resolution of head pain, radiofrequency ablation at C3, C4 and C5 that increased occipital pain, selective nerve block at $\mathrm{C} 2$ level that severely increased his pain, and multiple occipital nerve blocks that aggravated his pain. Prior medication trials had included divalproex and rofecoxib. Despite repeated adverse responses to occipital nerve blockade, the referring neurologist concurred with the prevailing diagnosis of occipital neuralgia and referred the patient for specialty head pain management.

At the head pain clinic, neurological examination revealed a healthy-appearing man reporting constant pain at the right lower occipital region, with radiating sharp pain in a right supraorbital distribution. The remainder of the neurological examination was entirely within normal limits. Further radiographic work-up included a brain MRI that showed several small foci of increased signal intensity in the white matter bilateral hemispheres that was felt to be a non-specific finding. Maxillofacial CT indicated moderate left ethmoid sinus mucosal thickening. The diagnosis was hemicrania continua or a possible secondary cervicogenic headache with medication overuse headache, with a recommendation for multidisciplinary treatment and admission to the facility's in-patient head pain unit.

The 16-day hospital course included an i.v. medication protocol (i.v. droperidol, diphenhydramine and magnesium); behavioural intervention; and multiple interventional procedures including a right suboccipital injection which worsened his pain, and a C2-C3 facet block which provided $24 \mathrm{~h}$ of head pain relief. The discharge medication regimen included: for head pain prevention, topiramate, quetiapine, verapamil; and to abort head pain, indomethacin, Midrin ${ }^{\circledR}$ and quetiapine. After discharge, the patient returned home where he underwent selective nerve blockade at $\mathrm{C} 0-\mathrm{C} 1$ and C1-C2 that provided moderate but temporary relief. He returned to the head pain clinic for radiofrequency ablation of the $\mathrm{C} 2-\mathrm{C} 3$ facet joint. This resulted in no appreciable change in his pain symptoms. At this point, the patient was then referred to physical therapy (J.K.R.) for examination for a possible musculoskeletal contribution to the head pain.

The physical therapy assessment noted ongoing constant level $2 / 5$ head pain ( 0 no pain at all, 5 severest pain) that increased to a severe level 5/5 two episodes per week. Activities that increased head pain included repetitive cervical motion, lifting his head while in left side-lying position and use of his right upper extremity. Previous courses of physical therapy and soft tissue massage had produced only temporary reduction in pain. Physical examination of posture and alignment revealed a slumped sitting posture. Range of motion (ROM) testing revealed restriction in right cervical rotation and cervical extension. Muscle tightness was noted in the right pectoralis muscle. His headache was reproduced with active motions including cervical extension and right upper extremity flexion. On pincher palpation of the right SCM, the most significant finding was immediate and dramatic reproduction of the patient's primary occipital and supraorbital pain. MTrPs were noted in the midmuscle belly superficial (sternal) head of the right SCM. The patient was assessed to have a primary myofascial pain involving the SCM, with contributing muscle imbalances and postural deviations. He volunteered that during previous evaluations by 
various practitioners, the SCM had not been palpated, which is needed to diagnose MTrPs. Following the examination, kinesiotaping, a taping technique designed to reduce underlying muscle tone, was utilized to reduce the tone of the SCM.

The patient was referred to a local physical therapist with specific instructions to reduce trigger point activation in the SCM. Therapeutic instructions included trigger point release with deep soft-tissue mobilization and manual therapy (e.g. muscle energy technique), stretching exercises, electromyographic (EMG) biofeedback specific for SCM relaxation, and sitting postural re-education to increase lumbar lordosis and reduce forward head position. Four days later, the patient phoned the clinic to report that kinesiotaping had reduced his pain by $50 \%$. Following 2 weeks of local therapies, the patient reported dramatic reduction in his pain. He had reduced his analgesic usage by two-thirds and was reporting that his pain was mild, never escalating to moderate or severe levels. He was able to perform activities of daily living including shopping and vacuuming without pain. He reported that his best headache days previously were now his worst days. At 4 weeks his physical therapist reported that his head pain rating had reduced by approximately $70 \%$. He was sleeping 5-6 h continuously and night awakenings were no longer due to pain. At 6 months' follow-up, phone reassessment indicated that since his discharge from a 6-week, 16-session course of physical therapy, he continued to be pain free approximately $1 / 3-1 / 2$ of the time with 'mild discomfort' the remainder of the time. He had had no episodes of restricted ROM or muscle spasm of the cervical musculature over the previous 3-4 months. He maintained adherence to a brief home stretching exercise programme and continued to use corrected posture to prevent discomfort.

\section{Discussion}

Forty years ago Travell (19) described the influence of biomechanical factors in the aetiology and maintenance of chronic headache, with particular reference to faulty posture and its effect on muscle strain and overload of the SCM, associated with MTrPs that caused head pain. Much has been written about myogenic headaches that derive from MTrPs $(10,20-22)$ and the various referred head pain patterns that result from MTrPs of the cervical musculature are now well described (10). Unfortunately, there has been longstanding debate on the contribution of MTrPs in the aetiology of acute and chronic musculoskeletal pain. Recently, Simons (10, $23,24)$ has proposed the integrated hypothesis theory to explain the basic mechanisms of referred pain that results from MTrPs. As a result, a consensus on the pathophysiology of chronic myofascial pain and its associated referred pain is now developing, and recent experimental investigations have provided preliminary support for this model (6, 25-27). Despite these advances, there continues to be a prevailing view among some pain practitioners that musculoskeletal dysfunction is rarely a cause of intractable pain (28), that has led to an undervaluation of myofascial pain as a source of persistent pain. The case described here illustrates that this holds equally true for myogenic head pain that is repeatedly dismissed as cervicogenic headache.

The diagnosis of cervicogenic headache can be a significant clinical challenge. In this case, there was significant symptom overlap between head pain secondary to SCM MTrPs (see Fig. 1) and the clinical features of hemicrania continua, including continuous and persistent one-sided head pain, nasal congestion and conjunctival injection. Of particular note was the presence of autonomic features that are well known to be associated with hemicrania continua (4) but are less well appreciated as common symptomatic findings for MTrPs in the sternal division of the SCM (10). In addition, diverse anatomical pathways can cause cervicogenic headache. This patient had undergone multiple medical examinations by several medical specialties as well as receiving five previous courses of physical therapy. It is noteworthy that despite the many previous practitioners, all had failed to examine the SCM as a possible source of pain. This is probably because individuals with head pain from the SCM do not report pain along the distribution of the muscle, but rather across the occipital, supraorbital and temporal regions of the head (10). The failure to consider referred pain in locating musculoskeletal pain complaints is common in clinical practice and can lead to diagnostic confusion and misdirected interventions $(8,10)$. This is not the case with visceral sources of referred pain such as cardiac and gallbladder disease. With knowledge of the various referred pain patterns associated with cervical MTrPs, the pain clinician is enabled to work within a framework that relates specific patterns of pain with their associated TrPs, leading to their identification in the troubled muscle or muscle groups.

Moreover, as this case illustrates, when cervicogenic headache secondary to chronic myofascial pain is effectively treated by a multimodal approach, 

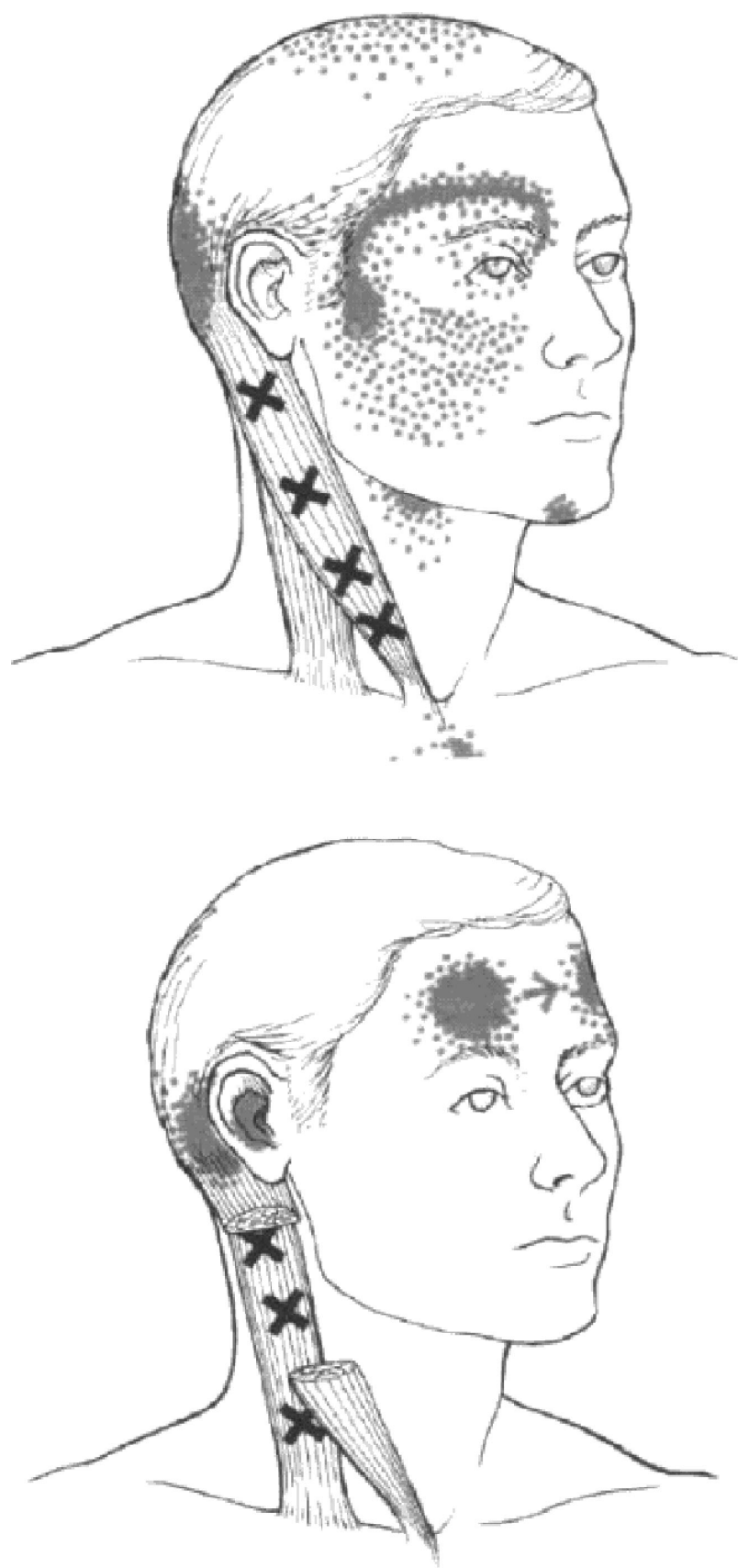

Figure 1 Referred pain patterns from common trigger points in the sternal (left) and clavicular (right) divisions of the sternocleidomastoid muscle. Reproduced, with permission, from: Simons DG, Travell JG, Simons LS. Travell and Simons' myofascial pain and dysfunction: the trigger point manual, Vol. 1, 2nd edn. Baltimore, MD: Williams \& Wilkins 1999: 310. (c) Lippincott Williams \& Wilkins.

there can be surprisingly rapid and prolonged diminution in clinical morbidity, even in longstanding cases $(19,20)$. The treatment of chronic myofascial pain requires a comprehensive approach directed to the underlying MTrP source, while simultaneously addressing the central importance of perpetuating factors that maintain $\operatorname{TrP}$ activation $(10,29)$. As noted, the physical therapy regimen prescribed was multifaceted, with attention directed to manual techniques to reduce $\operatorname{TrP}$ activity, including stretching to restore muscle length and elasticity, relaxation to decrease $\operatorname{Tr} \mathrm{P}$ activation and instruction to correct faulty postures. This report indicates that individual and isolated physical medicine modalities often fail to manage cervicogenic headache effectively, but that manual therapies combined with therapeutic exercise and posture re-education can produce significantly positive outcomes.

The SCM can be a particularly enigmatic and common source of myofascial cervicogenic headache (30). As the SCM does not overlie the posterior cervical spine, it is easily ignored during a physical examination for cervical dysfunctions that may underlie a headache and patients typically do not complain of neck pain with the presence of SCM MTrPs. SCM MTrPs and muscle hypertonicity are common sequelae of whiplash injuries from motor vehicle accidents (31-33) and whiplash injuries are a known and frequent initiating event for cervicogenic headaches (34). Forward-head posture can contribute to the development of SCM MTrPs. The SCM produces cervical flexion as its primary function. Compromised endurance of deep flexor muscle of the cervical spine is related to abnormal forward-head posture (35). There is also evidence that relative excessive EMG activity of the SCM, which could give rise to TrPs if recurrent, is related to weak deep cervical flexors (33). Several clinical studies have documented an association between deep cervical flexor weakness and cervicogenic headache $(33,36,37)$.

Anaesthetic nerve blockade to confirm the diagnosis of cervicogenic headache has become increasingly popular $(14,16)$. Anaesthetic cervical blocks can identify, at times, the level of spinal root mediation of pain, but not necessarily pinpoint the abnormal cervical abnormality generating the pain signal. Indeed, the clinical utility of nerve blocks has been challenged, based on their lack of diagnostic specificity and the absence of empirical evidence to confirm long-term therapeutic benefit (38-40). In this case, the patient had variable responses to multiple (often repeated) cervical blocks and no appreciable benefit from neurotomy of the C2-C3. In contrast, manual techniques, that include assessment of intervertebral segmental motion, muscle length and joint restrictions, have been found to be reliable and equal to facet blockade in confirming 
the level of facet dysfunction that mediates a cervicogenic headache (41). Moreover, careful clinical examination that emphasizes myogenic dysfunction may be more comprehensive and effective in identifying the range of cervical abnormalities that can produce head pain. This case report highlights the relative advantage of a hands-on physical examination, with careful palpation of the cervical musculature, when evaluating a patient with suspected cervicogenic headache.

\section{Acknowledgement}

The authors thank Todd Rozen MD for his very helpful comments on an earlier draft of this manuscript.

\section{References}

1 Bogduk N. Cervicogenic headache: anatomic basis and pathophysiological mechanisms. Curr Pain Headache Rep 2001; 5:382-6.

2 Fredriksen TA, Hovdal H, Sjaastad O. Cervicogenic headache: clinical manifestations. Cephalalgia 1987; 7:147-60.

3 Pollmann W, Keidel M, Pfaffenrath V. Headache and the cervical spine: a critical review. Cephalalgia 1997; 17: 1801-16.

4 International Headache Society. The International Classification of Headache Disorders, 2nd edition. Cephalalgia 2004; 24 (Suppl. 1):9-160.

5 Hoheisel U, Mense S, Simons DG, Yu X-M. Appearance of new receptive fields in rat dorsal horn neurons following noxious stimulation of skeletal muscle: a model for referred muscle pain? Neurosci Lett 1993; 153:9-12.

6 Mense S, Simons DG, Russell IJ. Muscle pain: understanding its nature, diagnosis and treatment. Baltimore: Lippincott Williams \& Wilkins 2001.

7 Fishbain DA, Lewis J, Cole B, Cutler RB, Rosomoff RS, Rosomoff HL. Do the proposed cervicogenic headache diagnostic criteria demonstrate specificity in terms of separating cervicogenic headache from migraine? Curr Pain Headache Rep 2003; 7:387-94.

8 Yi X, Cook AJ, Hamill-Ruth RJ, Rowlingson JC. Cervicogenic headache in patients with presumed migraine: missed diagnosis or misdiagnosis. J Pain 2005; 6:700-3.

9 Edmeads J. The cervical spine and headache. Neurology 1989; 38:1874-8.

10 Simons DG, Travell JG, Simons LS. Travell and Simons' myofascial pain and dysfunction: the trigger point manual, Vol. 1, 2nd edn. Baltimore: William \& Wilkins 1999.

11 Fernández-de-Ias-Penas C, Fernandez J, Miangolarra J. Musculoskeletal disorders of mechanical neck pain: myofascial trigger points versus cervical joint dysfunction-a clinical study. J Musculoskel Pain 2005; 13:27-35.

12 Fernández-de-Ias-Penas C, Alonso-Blanco C, Cuadrado ML, Gerwin R, Pareja JA. Trigger points in the subocciptal muscles and forward head posture in tension-type headache. Headache 2006; 46:454-60.

13 Sjaastad O, Fredricksen TA, Stolt-Nielsen A, Salvesan R, Jansen J, Pareja JA et al. Cervicogenic headache: a clinical review with special emphasis on therapy. Funct Neurol 1997; 12:305-17.

14 Bogduk N. Local anesthetic blocks of the second cervical ganglion: a technique with application in occipital headache. Cephalalgia 1981; 1:41-50.

15 Gawel MJ, Rothbart PJ. Occipital nerve block in the management of headache and cervical pain. Cephalalgia 1992; 12:9-13.

16 Bovim G, Berg R, Dale LG. Cervicogenic headache: anesthetic blockades of cervical nerves (C2-C5) and facet joint (C2/3). Pain 1992; 49:315-20.

17 Meloche JP, Bergeron Y, Bellavance A, Morand M, Huot J, Belzile G. Painful intervertebral dysfunction: Robert Maigne's original contribution to headache of cervical origin. Headache 1993; 33:328-34.

18 Zito G, Jull G, Story I. Clinical tests of musculoskeletal dysfunction in the diagnosis of cervicogenic headache. Manual Ther 2006; 11:118-29.

19 Travell J. Mechanical headache. Headache 1967; 7:23-9.

20 Davidoff RA. Trigger points and myofascial pain: toward understanding how they affect headaches. Cephalalgia 1998; 18:436-48.

21 Jaeger B. Are 'cervicogenic' headaches due to myofascial pain and cervical spine dysfunction? Cephalalgia 1989; 9:157-64.

22 Fernández-de-Ias-Penas C, Cuadrado ML, Gerwin R, Pareja JA. Referred pain from the trochlear region in tension-type headache: a myofascial trigger point from the superior oblique muscle. Headache 2005; 45:731-7.

23 Simons DG. Neurophysiological basis of pain caused by trigger points. Am Pain Soc J 1994; 3:17-9.

24 Simons DG. Review of enigmatic MTrPs as a common cause of enigmatic musculoskeletal pain and dysfunction. J Electromyog Kinesiol 2004; 14:95-107.

25 Gerwin RD, Dommerholt J, Shah J. An expansion of Simons' integrated hypothesis of trigger point formation. Curr Pain Headache Rep 2004; 8:468-75.

26 McPartland JM. Trigger points: molecular and osteopathic perspectives. J Am Osteopath Assoc 2004; 104:244-9.

27 Shah J, Phillips T, Danoff J, Gerber L. An in-vitro microanalytic technique for measuring the local biochemical milieu of human skeletal muscle. J Appl Physiol 2005; 99:1980-7.

28 Fordyce WE. Back pain in the workplace. Seattle: IASP Press 1995.

29 Graff-Radford SB, Reeves JL, Jaeger B. Management of chronic head and neck pain: effectiveness of altering factors perpetuating myofascial pain. Headache 1987; 27:186-90.

30 Cibulka MT. Sternocleidomastoid muscle imbalance in a patient with recurrent headache. Manual Ther 2006; 11:78-82.

31 Baker BA. The muscle trigger: evidence for overload injury. J Neurol Orthop Med Surg 1986; 7:35-43.

32 Kumar S, Narayan Y, Amell T. An electromyographic study of low-velocity rear-end impacts. Spine 2002; 27:1044-55.

33 Jull G, Kristjansson E, Dall'Alba P. Impairment in the cervical flexors: a comparison of whiplash and insidious onset neck pain patients. Manual Ther 2004; 9:89-94. 
34 Drottning M, Staff PH, Sjaastad O. Cervicogenic headache (CEH) after whiplash injury. Cephalalgia 2002; 22:16571.

35 Watson DH, Trott PH. Cervical headache: an investigation of natural head posture and upper cervical flexor muscle performance. Cephalalgia 1993; 13:272-84.

36 Jull G, Barrett C, Magee R, Ho P. Further clinical clarification of the muscle dysfunction in cervical headache. Cephalalgia 1999; 19:179-85.

37 Falla D, Jull GA, Hodges PW. Neck pain patients demonstrate reduced EMG activity of the deep cervical flexor muscles during performance of cranio-cervical flexion. Spine 2004; 29:2108-14.
38 Hogan QH, Abrams SE. Neural blockade for diagnosis and prognosis. Anesthesiology 1997; 86:216-41.

39 Sanders SH, Harden N, Vincente PJ. Evidence-based clinical practice guidelines for interdisciplinary rehabilitation of chronic nonmalignant pain syndrome patients. World Inst Pain 2005; 5:303-15.

40 Schwarzer AC, Aprill CN, Derby R, Fortin J, Kine G, Bogduk N. The false-positive rate of uncontrolled diagnostic blocks of the lumbar zygapophysial joints. Pain 1994; 58:195-200.

41 Jull G, Bogduk N, Marsland A. The accuracy of manual diagnosis for cervical zygapophysial joint pain syndromes. Med J Aust 1988; 148:233-6. 